\title{
Body Appreciation in Lesbian, Bisexual, and Queer Women: Examining a Model of Social Support, Resilience, and Self-Esteem
}

\author{
C. Blair Burnette, Melissa A. Kwitowski, Michael A. Trujillo, and Paul B. Perrin
}

\begin{abstract}
Purpose: There is increasing research on positive body image, but no studies to date have examined these constructs in lesbian, bisexual, and queer (LBQ) women. However, LBQ women are at increased risk for mental health concerns and disordered eating, and there is evidence that body appreciation might be both adaptive and protective. This study examined factors that could uniquely relate to body appreciation in LBQ women.

Method: Women identifying as LBQ $(N=150)$ completed demographics and measures of social support, resilience, self-esteem, and body appreciation. We tested a hypothesized mediational model of social support leading indirectly to body appreciation through resilience and self-esteem, controlling for body mass index.

Results: All direct effects, except social support to body appreciation $(p=0.696)$, were significant ( $p s=0.017-$ 0.001 ), reflecting a full multiple mediation. As hypothesized, the effect of social support on body appreciation was indirect ( $p=0.011)$, through resilience and self-esteem.

Conclusion: This is the first study to investigate factors that might facilitate positive body image in LBQ women. Although preliminary, results suggest social support, resilience, and self-esteem might be important targets of body image interventions with $L B Q$ women.
\end{abstract}

Keywords: body appreciation; women; sexual minority; social support; resilience; self-esteem

Body dissatisfaction is endemic among women in Western societies, and is related not only to disordered eating and eating disorders (EDs), but also smoking initiation, depression, suicidal ideation, binge eating, reduced fruit and vegetable intake, and sedentary behaviors. ${ }^{1-6}$ The role of sexual identity in body image is understudied and findings to date are mixed. Some studies suggest lesbian women experience less body dissatisfaction, ${ }^{7-9}$ which researchers posit could result from lesbian women being more likely to reject heteronormative beauty ideals and accept diverse body types. ${ }^{8,10,11}$ Conversely, in other studies, no significant difference in body dissatisfaction between lesbian and heterosexual women has been found. ${ }^{12-14}$
One theory for the lack of differences is that all women in Westernized cultures, regardless of sexual identity, are socialized to measure themselves by and conform to rigid beauty ideals that emphasize thinness and femininity. ${ }^{15}$ There is considerably less research on the experiences of women identifying as bisexual, pansexual, or queer. ${ }^{16-18}$ However, extant evidence confirms that body image is multidimensional, ${ }^{19}$ with sexual identity being the only one influential factor. Moreover, there is considerable within-group heterogeneity. For instance, degree of femininity/masculinity/androgyny, peer sexual orientation, partner choice, media representations, and fashion all seem to contribute to body image in lesbian, bisexual, and queer (LBQ) women. ${ }^{16-18}$ 
There is growing interest in positive body image as a protective factor against body dissatisfaction and its related consequences. ${ }^{20}$ Positive body image is multidimensional, and one facet, body appreciation, refers to an appreciation of one's body that extends beyond appearance. ${ }^{21}$ Body appreciation is related to adaptive media strategies (e.g., protective filtering), ${ }^{22}$ increased self-esteem, and proactive coping strategies, even when controlling for appearance dissatisfaction. ${ }^{21}$ Body appreciation might also reduce the effects of thin-ideal media exposure. ${ }^{23}$ Because of its adaptive nature and potential as a protective factor, it is an increasing target of intervention work..$^{24,25}$ Despite its promise, there is no known research examining body appreciation in LBQ women.

It is unclear if LBQ women experience less body dissatisfaction; however, disordered eating levels seem comparable, or even greater than, heterosexual women. ${ }^{26,27}$ Moreover, LBQ women are exposed to greater prejudice and discrimination, report greater mental health concerns, and report greater unmet mental health needs. ${ }^{28}$ Body image is an important component of mental health that is associated with a spectrum of mental and physical health outcomes. ${ }^{1}$ Thus, examining factors that facilitate positive body image in a group vulnerable to mental health concerns and inadequate mental health treatment is imperative.

The determinants of body image in LBQ women are complex. ${ }^{18}$ Some factors that might be uniquely related to body image in this group include social support, resilience, and self-esteem. The literature on the benefits of positive social support to physical and mental health is robust. ${ }^{29}$ Social support seems particularly important in the LBQ community as it is related to identity development and adjustment, and can buffer the effects of social stress and rejection. ${ }^{30-32}$ Positive social support is an important resource that strengthens resilience, the successful adaptation to challenging circumstances, particularly in the LBQ community. ${ }^{29,33}$ For instance, in a study of LBQ women who experienced family rejection, those with greater resilience had sought out and developed connections with other members of their LBQ communities. ${ }^{32}$ One way social support aids resilience in LBQ individuals is through lowering reactivity to prejudice, consistent with minority stress theory. ${ }^{34,35}$

Both positive social support and resilience are related to self-esteem. For instance, social support through minority community participation appears to promote an increased sense of both individual and collective self- esteem as a result of the affiliation with other minority individuals. ${ }^{32}$ Although research often examines selfesteem as a protective factor for resilience, resilience also seems to foster self-esteem, potentially through positive affect and self-efficacy. ${ }^{36,37}$ Self-esteem is closely tied to body image, particularly in women. ${ }^{38,39}$ There is scant research in LBQ women, but Striegel-Moore et al. ${ }^{12}$ found self-esteem was more strongly related to body esteem in lesbian than heterosexual women.

The purpose of this study was to examine factors that facilitate body appreciation in LBQ women. We hypothesized a multiple mediational model, where social support would be indirectly related to body appreciation through resilience and self-esteem (Fig. 1). We expected positive direct associations between social support and both resilience and self-esteem, resilience and both self-esteem and body appreciation, and selfesteem and body appreciation. We did not expect a significant direct association between social support and body appreciation after controlling for this series of mediators. Current body image interventions do not consider the role of sexual identity. This study will address a gap in the literature, and findings could inform the development and refinement of interventions for LBQ women.

\section{Method}

\section{Participants and procedure}

The host university's Institutional Review Board approved the study. Participants were recruited as part of a larger study on the mental and physical health of lesbian, gay, bisexual, transgender, and queer (LGBTQ) individuals. Recruitment occurred through Internet forums and groups and emails to national (i.e., United States) and regional (i.e., within the United States) LGBTQ organizations. The purpose of this study was described as an investigation of the physical and mental health of racial/ethnic minorities who identify as

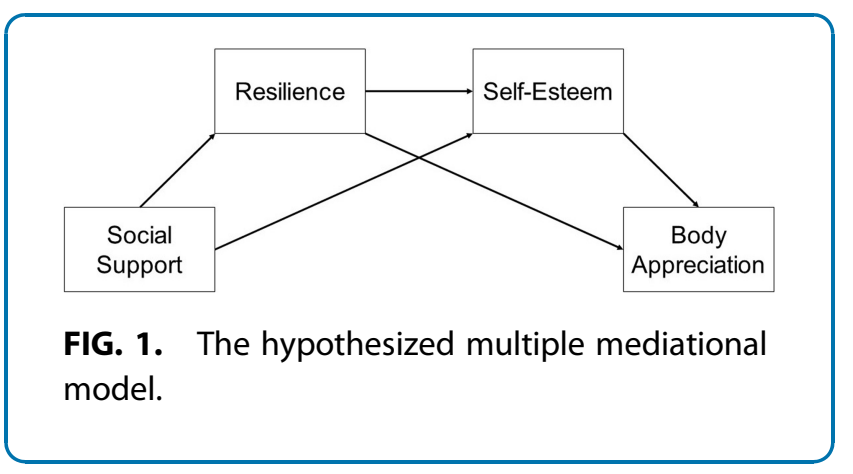


Table 1. Sample Characteristics

\begin{tabular}{|c|c|c|c|c|}
\hline & Bisexual $(n=58)$ & Lesbian $(n=49)$ & Queer $(n=43)$ & Overall $(N=150)$ \\
\hline Age (range: 18-66) & $31.53(10.11)$ & 37.09 (13.95) & $25.33(6.65)$ & $31.90(11.95)$ \\
\hline \multicolumn{5}{|l|}{ Race/ethnicity } \\
\hline White/European American & $34.7 \%(17)$ & $31.0 \%(18)$ & $20.9 \%(9)$ & $29.3 \%(44)$ \\
\hline Black/African American & $22.4 \%(11)$ & $25.9 \%(15)$ & $30.2 \%(13)$ & $26.0 \%(39)$ \\
\hline Asian/Pacific Islander & $8.2 \%(4)$ & $13.8 \%(8)$ & $30.2 \%(13)$ & $16.7 \%(25)$ \\
\hline Multiracial & $18.4 \%(9)$ & $10.3 \%(6)$ & $9.3 \%(4)$ & $12.7 \%(19)$ \\
\hline Latina & $12.2 \%(6)$ & $13.8 \%(8)$ & $7.0 \%(3)$ & $11.3 \%(17)$ \\
\hline American Indian & $2.0 \%(1)$ & $3.4 \%(2)$ & $2.3 \%(1)$ & $2.7 \%(4)$ \\
\hline Other & $2.0 \%(1)$ & $1.7 \%(1)$ & - & $1.3 \%(2)$ \\
\hline BMI & $27.34(6.91)$ & $27.63(7.95)$ & 30.67 (10.91) & $28.41(8.67)$ \\
\hline$<18.5$ & $2.0 \%(1)$ & $6.9 \%(4)$ & $4.7 \%(2)$ & $4.7 \%(7)$ \\
\hline $18.5-24.9$ & $49.0 \%(24)$ & $43.1 \%(25)$ & $34.9 \%(15)$ & $42.7 \%(64)$ \\
\hline $25-29.9$ & $18.4 \%(9)$ & $17.2 \%(10)$ & $23.3 \%(10)$ & $19.3 \%(29)$ \\
\hline$\geq 30$ & $30.6 \%(15)$ & $32.8 \%(19)$ & $37.2 \%(16)$ & $33.3 \%(50)$ \\
\hline SS (range: 12-60) & $45.18(9.16)$ & 44.93 (10.57) & $43.77(10.35)$ & $44.68(10.01)$ \\
\hline BRS (range: 6-30) & $18.37(4.81)$ & $19.12(5.60)$ & $17.26(5.07)$ & $18.34(5.22)$ \\
\hline RSES (range: $0-30$ ) & $17.94(5.71)$ & $19.57(5.60)$ & $16.35(6.74)$ & $18.11(6.09)$ \\
\hline BAS (range: $1.23-4.92$ ) & $3.36(0.72)$ & $3.52(0.66)$ & $3.28(0.90)$ & $3.40(0.76)$ \\
\hline
\end{tabular}

Note: Continuous variables are presented as M(SD). Categorical variables are presented as $\%(n)$.

BAS, Body Appreciation Scale; BMI, body mass index; BRS, Brief Resilience Scale; RSES, Rosenberg Self-Esteem Scale; SS, social support.

LGBTQ. Interested individuals emailed the research coordinator who screened for eligibility (i.e., at least 18 years old and identifying as a sexual minority woman). Eligible individuals accessed the online survey through a link and access code sent through email. Electronic consent was obtained. Participants received a $\$ 15$ gift card upon study completion.

The sample $(N=150)$ identified as $32.7 \%$ bisexual ( $n=49), 38.7 \%$ gay/lesbian $(n=58)$, and $28.6 \%$ queer/ other $(n=43)$. Five of the six women identifying as "other" described their sexual orientation as pansexual. Women ranged between 18 and 66 years $(M=31.90$, $S D=11.95)$. The sample was fairly racially/ethnically diverse, with $29.3 \%$ White $(n=44), 26.0 \%$ Black $(n=39)$, 16.7\% Asian/Hawaiian/Pacific Islander $(n=25), 12.7 \%$ multiracial $(n=19), 11.3 \%$ Latinx $(n=17), 2.7 \%$ American Indian $(n=4)$, and $1.3 \%$ "other" $(n=2)$.

Six percent of the sample $(n=9)$ had received their high school diploma or General Education Development Certificate, $24.7 \%(n=37)$ had attended some college without a degree, 9.3\% $(n=14)$ had a 2 -year degree, $30.0 \%(n=45)$ had a 4 -year degree, $22.0 \%$ $(n=33)$ had a master's degree, and $8.0 \%(n=12)$ had a doctorate. Forty-four percent of the sample $(n=66)$ reported being employed full-time, $12.0 \% \quad(n=18)$ part-time, $18 \%(n=27)$ were both employed and a student, $16.0 \%(n=24)$ were students, and $10.0 \%(n=15)$ were unemployed. The majority of the sample reported being in some type of romantic relationship, with $42.7 \%$ $(n=64)$ reporting a long-term ( $>12$ months) monogamous relationship, $13.3 \%(n=20)$ a new ( $<12$ months) monogamous relationship, $14.0 \%(n=21)$ dating more than one person, and $30.0 \%(n=45)$ not currently dating or in a relationship. See Table 1 for participant characteristics displayed by sexual orientation and overall.

\section{Measures}

Demographics. Participants were surveyed on their gender, sexual, and racial/ethnic identities, age, height, weight, social class, education level, and employment and relationship status.

Body Appreciation Scale. The Body Appreciation Scale $(\mathrm{BAS})^{40}$ is a 13-item measure of an individual's body appreciation, acceptance, and respect. Items are rated from $1=$ Never to $5=$ Always and averaged to derive a total score, with higher scores reflecting greater body appreciation. The BAS was updated after data collection; nevertheless, the original BAS demonstrated good internal consistency, reliability, and construct validity with college women. ${ }^{41}$ Cronbach's alpha in this study was 0.92.

Rosenberg Self-Esteem Scale. The Rosenberg SelfEsteem Scale (RSES) ${ }^{42}$ contains 10 items, 5 negatively worded, which measure positive self-evaluations. Items are rated $1=$ Strongly Disagree to $4=$ Strongly Agree. In this study, negatively worded items were reverse coded and items were summed to derive an overall score. Higher scores indicate greater self-esteem. Cronbach's alpha was 0.92 .

Brief Resilience Scale. The Brief Resilience Scale $(B R S)^{43}$ measures the ability to recover from stress, 
and is not specific to sexual or gender identity. Six items are rated from $1=$ Strongly Disagree to $5=$ Strongly Agree, with three reverse coded. An example item states, "I tend to bounce back quickly after hard times." The total score represents the sum of item responses, with higher scores reflecting greater resilience. Cronbach's alpha was 0.88 .

Multidimensional Scale of Perceived Social Support. The Multidimensional Scale of Perceived Social Support (MSPSS ${ }^{44}$ assesses the subjective impression of social support adequacy in three specific domains: friends, family, and significant others. Twelve items are rated from $1=$ Strongly Disagree to $5=$ Strongly Agree. Total scores represent the sum of responses, with higher scores indicating greater perceived support. Cronbach's alpha was 0.91 .

\section{Data analysis}

Preliminary analyses. Participant data were deleted from the survey software if there was an indication of impossible response patterns (e.g., selecting the first response for every single item), responses from a computer (i.e., $<20 \mathrm{~min}$ or $>24 \mathrm{~h}$ to complete), or if participants did not correctly respond to four of six (66.6\%) randomly placed validation questions (e.g., "Please select strongly agree for the item below"). Assumptions of planned analyses, including normality, and univariate and multivariate outliers, were assessed. Descriptive statistics and bivariate correlations between study variables were conducted using SPSS Version 25.0. ${ }^{45} \mathrm{We}$ examined the associations between age, sexual orientation, race/ethnicity, body mass index (BMI), and primary study variables, and significant associations were considered as covariates. One-way between-subjects analyses of variance explored differences on age, BMI, and outcome variables across sexual orientation (i.e., bisexual, lesbian, and queer). Significant omnibus tests were followed by Tukey post hoc comparisons to locate differences. We judged significance at $\alpha=0.01$ to account for multiple comparisons.

Path analysis. A mediational path model was developed using $\mathrm{AMOS}^{46}$ to examine a hypothesized series of connections leading from social support to body appreciation. See Figure 1 for the hypothesized model. Direct and indirect effects were estimated using 2000 bootstrap samples and bias-corrected $95 \%$ confidence intervals. Because the sample size in this study was lower than the 200 recommended by Boomsma and Hoogland, ${ }^{47}$ fit indices were omitted as they would likely be more obscuring than illuminating. Instead, the focus was on direct and indirect effects. However, our sample size exceeded the acceptable ratio of 10 cases for each free parameter, ${ }^{48}$ supporting the appropriateness of path analysis. Because extant research suggests that body image might differ across sexual orientation, ${ }^{18}$ we ran the model overall and separately by sexual orientation. However, because the small group sizes reduced power to detect effects, these analyses were exploratory in nature.

\section{Results}

\section{Preliminary analyses}

Skewness and kurtosis for all variables were normal (coefficients \pm 1.5 ). Z-scores were $<3.0 \mathrm{SDs}$ and visual inspection of scatterplots did not reveal any outliers. ${ }^{49}$ There was a significant negative correlation $(r=-0.21$, $p=0.011$ ) between BMI and body appreciation; thus, BMI was included as a covariate. See Table 1 for descriptive statistics. There were no significant group differences on study variables, except for age. A post hoc Tukey test revealed that the queer sample was significantly younger than the lesbian sample $(p<0.001)$.

\section{Mediational path model}

A path model examined direct and indirect effects of social support on body appreciation through resilience and self-esteem, controlling for BMI. See Table 2 for an overview of direct and indirect effects. To control for BMI, a path was drawn from BMI to body appreciation. In the first model, we drew paths from social support to resilience, self-esteem, and body appreciation; from resilience to self-esteem and body appreciation; and from self-esteem to body appreciation. As hypothesized, the path from social support to body appreciation was not significant $(\beta=-0.022, S E=0.071, p=$ 0.696). We trimmed the nonsignificant path and reran the model. Because direct and indirect effect estimates remained largely unchanged, we present results only for the final model.

When controlling for BMI, greater social support was related to greater resilience $(p=0.001)$ and higher self-esteem $(p=0.002)$. Similarly, greater resilience was related to both higher self-esteem $(p=0.001)$ and body appreciation $(p=0.017)$, and higher selfesteem was positively associated with body appreciation $(p=0.001)$. All indirect effects were significant, including social support on self-esteem through resilience $(p=0.001)$, resilience on body appreciation through self-esteem $(p=0.001)$, and social support 
Table 2. Bootstrapped, Standardized Estimates of the Multiple Mediational Path Analysis

\begin{tabular}{|c|c|c|c|c|c|c|c|c|}
\hline & \multicolumn{2}{|c|}{ Bisexual $(n=49)$} & \multicolumn{2}{|c|}{ Lesbian $(n=58)$} & \multicolumn{2}{|c|}{ Queer $(n=43)$} & \multicolumn{2}{|c|}{ Total sample $(N=150)$} \\
\hline & $\beta(S E)$ & $p$ & $\beta(S E)$ & $p$ & $\beta(S E)$ & $p$ & $\beta(S E)$ & $p$ \\
\hline \multicolumn{9}{|l|}{ Direct effects } \\
\hline $\mathrm{BMI} \rightarrow \mathrm{BAS}$ & $-\mathbf{0 . 3 4 0}(0.104)$ & 0.002 & $-0.140(0.091)$ & 0.149 & $-0.197(0.113)$ & 0.065 & $-0.226(0.061)$ & 0.002 \\
\hline $\mathrm{SS} \rightarrow \mathrm{BRS}$ & $\mathbf{0 . 2 9 2}(0.109)$ & 0.018 & $0.509(0.108)$ & 0.001 & $0.266(0.153)$ & 0.097 & $0.382(0.074)$ & 0.001 \\
\hline $\mathrm{SS} \rightarrow \mathrm{RSES}$ & $0.291(0.148)$ & 0.084 & $0.206(0.113)$ & 0.104 & $0.319(0.121)$ & 0.017 & $0.268(0.074)$ & 0.002 \\
\hline $\mathrm{BRS} \rightarrow \mathrm{RSES}$ & $0.277(0.137)$ & 0.077 & $0.571(0.092)$ & 0.001 & $0.445(0.119)$ & 0.005 & $0.445(0.069)$ & 0.001 \\
\hline $\mathrm{BRS} \rightarrow \mathrm{BAS}$ & $\mathbf{0 . 3 4 5}(0.117)$ & 0.008 & $-0.083(0.131)$ & 0.541 & $0.138(0.130)$ & 0.240 & $0.180(0.076)$ & 0.017 \\
\hline RSES $\rightarrow$ BAS & $0.176(0.150)$ & 0.305 & $\mathbf{0 . 6 8 3}(0.106)$ & 0.001 & $0.579(0.110)$ & 0.002 & $0.458(0.082)$ & 0.001 \\
\hline \multicolumn{9}{|l|}{ Indirect effects } \\
\hline $\mathrm{SS} \rightarrow \mathrm{RSES}$ & $\mathbf{0 . 0 8 1}(0.055)$ & 0.044 & $0.291(0.081)$ & $<0.001$ & $0.118(0.080)$ & 0.059 & $0.170(0.043)$ & 0.001 \\
\hline $\mathrm{BRS} \rightarrow \mathrm{BAS}$ & $0.049(0.056)$ & 0.222 & $\mathbf{0 . 3 9 0}(0.097)$ & 0.001 & $0.258(0.092)$ & 0.004 & $0.204(0.054)$ & 0.001 \\
\hline $\mathrm{SS} \rightarrow \mathrm{BAS}$ & $0.166(0.079)$ & 0.018 & $0.297(0.090)$ & 0.001 & $0.290(0.095)$ & 0.004 & $0.269(0.053)$ & 0.011 \\
\hline
\end{tabular}

Parameters significant at $p<0.05$ are given in bold.

on body appreciation through resilience and selfesteem $(p=0.011)$. That is, social support was not directly associated with body appreciation; rather, the effect was indirect, with greater social support being related to both greater resilience and higher self-esteem, which contributed to higher body appreciation in the sample. See Figure 2 for the final model presented with standardized regression coefficients.

Group differences exploratory analyses. We ran the final model separately for bisexual, lesbian, and queer women. See Table 2 for an overview of direct and indirect effects across groups. In bisexual women, the association between BMI and body appreciation $(\beta=-0.340)$ was stronger than in lesbian $(\beta=-0.140)$ and queer women $(\beta=-0.226)$. Resilience was also more strongly related to body appreciation in the bisexual sample $(\beta=0.345)$ than lesbian $(\beta=-0.083)$ and queer $(\beta=$

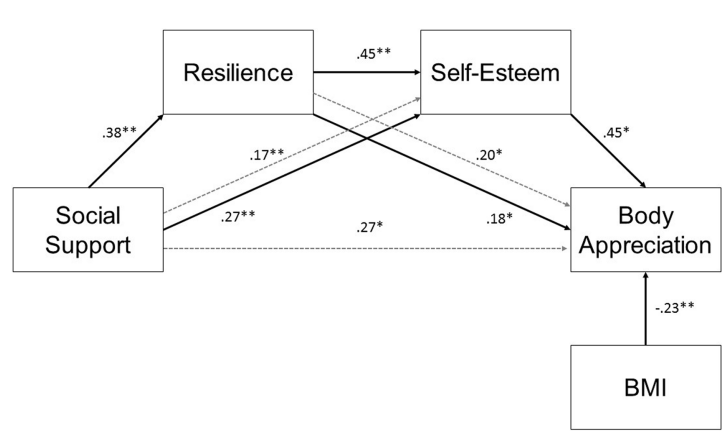

FIG. 2. The final multiple mediational model with standardized regression coefficients. Indirect effects are represented by dashed arrows. ${ }^{*} p<0.05 ;{ }^{* *} p<0.01$. BMl, body mass index.
0.138) samples. Conversely, associations with self-esteem were generally weaker in bisexual women. For instance, resilience displayed stronger relations with self-esteem in lesbian $(\beta=0.571)$ and queer women $(\beta=0.445)$ than bisexual women $(\beta=0.277)$. Similarly, self-esteem related more strongly to body appreciation in lesbian $(\beta=.683)$ and queer women $(\beta=0.579)$ than bisexual women $(\beta=0.176)$. Finally, the multiple mediation effect was stronger in the lesbian $(\beta=0.297)$ and queer samples $(\beta=0.290)$ relative to the bisexual sample $(\beta=0.166)$. The other notable difference that emerged was in lesbian women, where social support related more strongly to resilience $(\beta=0.509)$ than in bisexual $(\beta=0.292)$ and queer women $(\beta=0.266)$.

\section{Discussion}

This study examined a multiple mediational model of body appreciation in LBQ women. We hypothesized that social support would be indirectly related to body appreciation through resilience and self-esteem. We controlled for BMI, as it was significantly associated with body appreciation in our sample. Even when controlling for BMI, our hypothesized paths were significant. Consistent with previous research, perceived social support was related to both greater resilience and self-esteem overall. ${ }^{32,35,36}$ Thus, LBQ women in our sample who perceived they had positive support from family, friends, and others reported a greater ability to bounce back from stress and higher self-esteem. Resilience and self-esteem were also related in our sample, such that those reporting more resilience also reported higher self-esteem. In our model, both resilience and self-esteem were related to greater body appreciation. There is substantial evidence that selfesteem is related negatively to body dissatisfaction, ${ }^{38,39}$ 
and some evidence suggests it relates positively to body appreciation. ${ }^{40}$ Previous work has generally not considered the role of sexual identity. Thus, our study offers preliminary evidence that self-esteem is also associated with body appreciation in LBQ women.

As hypothesized, there was no significant direct association between perceived social support and body appreciation after controlling for resilience and selfesteem. Thus, perceived social support was related to body appreciation indirectly, through resilience and self-esteem, reflecting a full multiple mediation. In our sample, it appears that social support is a resource that aids LBQ women's successful adaption to stressful circumstances. Moreover, social support seems to be a source of self-esteem for this group, possibly through identification with a supportive and accepting community. ${ }^{32}$ Greater resilience was also related to self-esteem; previous research suggests that positive affect contributes to this association. ${ }^{36}$ Although not measured in this study, it is also plausible that resilience increases one's self-efficacy, thereby bolstering self-esteem. Of interest, both resilience and self-esteem were related to body appreciation in this group. It could be that perceived social support was a resource that strengthened resilience in our sample, helping participants cope with stressors such as discrimination, narrow societal beauty ideals, and weight stigma, which fostered self-worth and appreciation for their bodies.

Because research suggests body image differs across sexual identity, ${ }^{18}$ we ran the final model separately for bisexual, lesbian, and queer women as an exploratory analysis. However, these results should be interpreted with caution, given the small group sizes. Standard errors were larger than in the overall model, indicating lower reliability of parameter estimates, as would be expected with smaller samples. The most notable differences appeared between bisexual women relative to lesbian and queer women. For instance, BMI was more strongly associated with body appreciation in bisexual than lesbian or queer women. There is evidence that bisexual women are vulnerable to heightened body image concerns relative to lesbian women, in part because relationships with heterosexual men can encourage internalization of societal thinness ideals. ${ }^{50}$ Bisexual women in larger bodies might feel more pressure to conform to these ideals, and consequently have lower body appreciation.

Resilience and body appreciation displayed the strongest association in the bisexual sample. It is possible that bisexual women who feel they are successfully navigating the norms and pressures from both the het- erosexual and gay communities experience a greater appreciation for their bodies. The direct effects of self-esteem on social support, resilience, and body appreciation were relatively weaker in bisexual women compared with the other groups, as was the indirect effect of resilience on body appreciation through selfesteem. It appears that self-esteem was less relevant to body appreciation for bisexual women in this study. Previous research has found that bisexual women have lower self-esteem than heterosexual and gay women. ${ }^{51}$ Although self-esteem did not significantly differ between groups in this study, it is possible the determinants of self-esteem in bisexual women are more complex.

Despite some of these intergroup differences, full multiple mediation was found in each group, providing support for the validity of the model. This indicates that social support is related to increased resilience and selfesteem, which contribute to greater body appreciation in LBQ women. Body appreciation offers promise as a protective factor against harmful media effects and low selfesteem. $^{21,23}$ In addition, body dissatisfaction is a robust risk factor for disordered eating and $\mathrm{EDs},{ }^{5}$ which have considerable mental and physical health consequences. ${ }^{52}$ Thus, fostering positive body image in women who are both vulnerable to disordered eating ${ }^{27}$ and less likely to receive adequate treatment ${ }^{28}$ could be protective.

Results could be used both clinically and to inform future work. For instance, clinicians working with LBQ women could encourage their clients to build and strengthen their social networks, especially within the LBQ community. ${ }^{30}$ Clinicians might also help their clients process how they overcame difficult circumstances, which could foster a sense of resiliency. There are promising and brief interventions, such as an expressive writing program, with demonstrated efficacy to increase body appreciation ${ }^{24}$; however, these have not been tested or adapted specifically for LBQ women. Future work should assess whether existing interventions are appropriate for LBQ women, and consider incorporating themes of social support, resilience, and selfesteem to potentially improve outcomes for this group.

Positive body image research to date has not examined sexual identity. This is the first known study to examine factors related to body appreciation in LBQ women. Thus, this study is novel and offers preliminary evidence of factors that support positive body image in LBQ women. However, it has limitations. Self-report measures can result in biased and socially desirable responses. We assessed general resilience, 
rather than resilience specific to sexual orientationrelated discrimination or stigma. However, resilience reflects both a response to adversity and positive adaptation to the daily challenges and stressors of life, ${ }^{53}$ which can buffer the effects of stress, particularly for those exposed to stigma/discrimination. ${ }^{35}$ Given the documented health disparities in LGBT individuals ${ }^{54,55}$ it seems particularly important to identify protective factors, such as general resilience, self-esteem, and body appreciation, that could foster physical and mental health. Our sample was age diverse, and women identifying as queer were significantly younger than those identifying as lesbian. However, age was not significantly associated with, and groups did not significantly differ on, the outcome variables. It is possible that the small sample size precluded the ability to detect the nuanced effects of age in this study. As noted, group differences should be interpreted with caution given the small group sizes. However, because some paths appeared to differ between groups, future work should prioritize the recruitment of samples large enough to detect the unique contributors to body appreciation in each group. Data were collected before revision of the BAS. Nevertheless, the original measure displayed acceptable psychometrics in previous samples of women, ${ }^{41}$ and in this study. Finally, this study was cross-sectional. Future research should assess this model longitudinally, to investigate the temporal order of these relations.

\section{Conclusion}

This study offers preliminary evidence that social support, resilience, and self-esteem help foster body appreciation in LBQ women. This is important, as body appreciation might be protective against mental health concerns and disordered eating, which are elevated in LBQ women. To date, the most empirically supported body image interventions are neutral regarding sexual identity; however, these results suggest LBQ women might benefit from interventions that consider sexual identity and target factors uniquely relevant for this group, such as social support, resilience, and self-esteem.

\section{Acknowledgments}

The survey software for this study was funded by award number UL1TR000058 from the National Center for Research Resources. All ethical guidelines were followed in writing this article.

\section{Author Disclosure Statement}

No competing financial interests exist.

\section{References}

1. Bucchianeri MM, Neumark-Sztainer DR. Body dissatisfaction: an overlooked public health concern. J Ment Health. 2014;13:64-69.

2. Stice $E$, Shaw $H$. Prospective relations of body image, eating, and affective disturbances to smoking onset in adolescent girls: how Virginia slims. J Consult Clin Psychol. 2003;71:129-135.

3. Crow SJ, Eisenberg ME, Story $M$, et al. Suicidal behavior in adolescents: relationship to weight status, weight control behaviors, and body dissatisfaction. Int J Eat Disord. 2008;41:82-87.

4. Neumark-Sztainer DR, Paxton SJ, Hannan PJ, et al. Does body satisfaction matter? Five-year longitudinal associations between body satisfaction and health behaviors in adolescent females and males. J Adolesc Health. 2006;39:244-251.

5. Stice E, Shaw HE. Role of body dissatisfaction in the onset and maintenance of eating pathology. J Psychosom Res. 2002;53:985-993.

6. Rodin J, Silberstein L, Striegel-Moore RH. Women and weight: A normative discontent. In: Nebraska Symposium on Motivation., 1984: Psychology and Gender, Volume 32. Edited by Sonderegger TB. Lincoln, NE: University of Nebraska Press, 1984, pp. 267-307.

7. Bergeron SM, Senn CY. Body image and sociocultural norms: a comparison of heterosexual and lesbian women. Psychol Women Q. 1998;22:385-401.

8. Lakkis J, Ricciardelli LA, Williams RJ. Role of sexual orientation and gender-related traits in disordered eating. Sex Roles. 1999;41:1-16.

9. Alvy LM. Do lesbian women have a better body image? Comparisons with heterosexual women and model of lesbian-specific factors. Body Image. 2013;10:524-534.

10. Brown LS. Lesbians, weight and eating: new analyses and perspectives. In: Lesbian Psychologies: Explorations and Challenges. Edited by Collective BLP. Chicago: University of Illinois Press, 1987, pp. 294-309.

11. Swami V, Tovée MJ. The influence of body mass index on the physical attractiveness preferences of feminist and nonfeminist heterosexual women and lesbians. Psychol Women Q. 2006;30:252-257.

12. Striegel-Moore RH, Tucker N, Hsu J. Body image dissatisfaction and disordered eating in lesbian college students. Int J Eat Disord. 1990;9: 493-500.

13. Beren $\mathrm{SE}$, Hayden HA, Wilfley DE, et al. The influence of sexual orientation on body dissatisfaction in adult men and women. Int J Eat Disord. 1996;20:135-141.

14. Koff $E$, Lucas $M$, Migliorini R, et al. Women and body dissatisfaction: does sexual orientation make a difference? Body Image. 2010;7:255-258

15. Dworkin SH. Not in a man's image: lesbians and cultural oppression of body image. Women Ther. 1989;8:27-39.

16. Huxley CJ, Clarke V, Halliwell E. A qualitative exploration of whether lesbian and bisexual women are "protected" from sociocultural pressure to be thin. J Health Psychol. 2014;19:273-284.

17. Ludwig MR, Brownell KD. Lesbians, bisexual women, and body image: an investigation of gender roles and social group affiliation. Int J Eat Disord. 1999;25:89-97.

18. Smith ML, Telford E, Tree JJ. Body image and sexual orientation: the experiences of lesbian and bisexual women. J Health Psychol. 2017: 135910531769448.

19. Cash TF. Body image: past, present, and future. Body Image. 2004;1:1-5.

20. Tylka TL, Wood-Barcalow NL. What is and what is not positive body image? Conceptual foundations and construct definition. Body Image. 2015;14:118-129.

21. Tylka TL, Wood-Barcalow NL. The Body Appreciation Scale-2: item refinement and psychometric evaluation. Body Image. 2015;12:53-67.

22. Holmqvist K, Frisén A. "I bet they aren't that perfect in reality": appearance ideals viewed from the perspective of adolescents with a positive body image. Body Image. 2012;9:388-395.

23. Andrew R, Tiggemann M, Clark L. The protective role of body appreciation against media-induced body dissatisfaction. Body Image. 2015;15: 98-104.

24. Alleva JM, Martijn C, Van Breukelen GJP, et al. Expand Your Horizon: a programme that improves body image and reduces self-objectification by training women to focus on body functionality. Body Image. 2015;15:81-89.

25. Halliwell $E$, Jarman $H$, McNamara A, et al. Dissemination of evidencebased body image interventions: a pilot study into the effectiveness of using undergraduate students as interventionists in secondary schools. Body Image. 2015;14:1-4. 
26. Watson RJ, Adjei J, Saewyc E, et al. Trends and disparities in disordered eating among heterosexual and sexual minority adolescents. Int J Eat Disord. 2017;50:22-31.

27. Calzo JP, Austin SB, Micali N. Sexual orientation disparities in eating disorder symptoms among adolescent boys and girls in the UK. Eur Child Adolesc Psychiatry. 2018;27:1483-1490.

28. Burgess D, Lee R, Tran A, et al. Effects of perceived discrimination on mental health and mental health services utilization among gay, lesbian bisexual, and transgender persons. J LGBT Health Res. 2007;3:1-14.

29. Ozbay F, Johnson DC, Dimoulas $E$, et al. Social support and resilience to stress: from neurobiology to clinical practice. Psychiatry (Edgmont). 2007 $4: 35-40$.

30. Snapp SD, Watson RJ, Russell ST, et al. Social support networks for LGBT young adults: low cost strategies for positive adjustment. Fam Relat. 2015;64:420-430.

31. Friedman CK, Morgan EM. Comparing sexual-minority and heterosexual young women's friends and parents as sources of support for sexual issues. J Youth Adolesc. 2008;38:920-936.

32. Zimmerman L, Darnell DA, Rhew IC, et al. Resilience in community: a social ecological development model for young adult sexual minority women. Am J Community Psychol. 2015;55:179-190.

33. Ozbay F, Fitterling $H$, Charney D, et al. Social support and resilience to stress across the life span: a neurobiologic framework. Curr Psychiatry Rep. 2008;10:304-310.

34. Meyer IH. Prejudice, social stress, and mental health in lesbian, gay, and bisexual populations: conceptual issues and research evidence. Psychol Bull. 2003;129:674-697.

35. Kwon P. Resilience in lesbian, gay, and bisexual individuals. Personal Soc Psychol Rev. 2013;17:371-383.

36. Benetti C, Kambouropoulos N. Affect-regulated indirect effects of trait anxiety and trait resilience on self-esteem. Pers Individ Dif. 2006;41:341-352.

37. Kidd S, Shahar G. Resilience in homeless youth: the key role of selfesteem. Am J Orthopsychiatry. 2008;78:163-172.

38. Mellor D, Fuller-Tyszkiewicz M, McCabe MP, et al. Body image and selfesteem across age and gender: a short-term longitudinal study. Sex Roles. 2010;63:672-681.

39. Grossbard JR, Lee CM, Neighbors C, et al. Body image concerns and contingent self-esteem in male and female college students. Sex Roles. 2009;60:198-207.

40. Avalos L, Tylka TL, Wood-Barcalow N. The Body Appreciation Scale: development and psychometric evaluation. Body Image. 2005;2:285-297.

41. Tylka TL. Evidence for the Body Appreciation Scale's measurement equivalence/invariance between U.S. college women and men. Body Image. 2013;10:415-418.

42. Rosenberg M. Conceiving the Self. New York: Basic Books, 1979.

43. Smith BW, Dalen J, Wiggins $\mathrm{K}$, et al. The brief resilience scale: assessing the ability to bounce back. Int J Behav Med. 2008;15:194-200.
44. Zimet GD, Dahlem NW, Zimet SG, et al. The multidimensional scale of perceived social support. J Pers Assess. 1988;52:30-41.

45. IBM SPSS Statistics for Windows, Version 25.0. 2017. Armonk, NY: IBM Corporation.

46. Arbuckle JL. Amos., Version 23.0. 2014. Chicago, IL: IBM SPSS.

47. Boomsma A, Hoogland JJ. The robustness of LISREL modeling revisted. In: Structural Equation Modeling: Present and Future. Edited by Cudeck R, du Toit S, Sorbom D. Chicago, IL: Scientific Software International, 2001, pp. 139-168.

48. Bentler PM, Chou C-P. Practical issues in structural modeling. Sociol Methods Res. 1987;16:78-117.

49. Tabachnick BG, Fidell LS. Using Multivariate Statistics, 6th ed. Boston: Pearson, 2013.

50. Chmielewski JF, Yost MR. Psychosocial influences on bisexual women's body image. Psychol Women Q. 2013;37:224-241.

51. Yean C, Benau EM, Dakanalis A, et al. The relationship of sex and sexual orientation to self-esteem, body shape satisfaction, and eating disorder symptomatology. Front Psychol. 2013;4:887.

52. Kärkkäinen $U$, Mustelin $L$, Raevuori $A$, et al. Do disordered eating behaviours have long-term health-related consequences? Eur Eat Disord Rev. 2018;26:22-28.

53. Fletcher D, Sarkar M. Psychological resilience: a review and critique of definitions, concepts, and theory. Eur Psychol. 2013;18:12-23.

54. Fredriksen-Goldsen KI, Kim H-J, Bryan AEB, et al. The cascading effects of marginalization and pathways of resilience in attaining good health among LGBT older adults. Gerontologist. 2017;57(suppl 1):S72-S83.

55. Case $P$, Austin SB, Hunter DJ, et al. Sexual orientation, health risk factors, and physical functioning in the Nurses' Health Study II. J Women's Heal. 2004;13:1033-1047.

Cite this article as: Burnette $C B$, Kwitowski MA, Trujillo MA, Perrin PB (2019) Body appreciation in lesbian, bisexual, and queer women: examining a model of social support, resilience, and self-esteem, Health Equity 3:1, 238-245, DOI: 10.1089/heq.2019.0003.

Abbreviation Used

BAS $=$ Body Appreciation Scale

$\mathrm{BMI}=$ body mass index

$\mathrm{BRS}=$ Brief Resilience Scale

$\mathrm{LBQ}=$ lesbian, bisexual, and queer

LGBTQ = lesbian, gay, bisexual, transgender, and queer

MSPSS $=$ Multidimensional Scale of Perceived Social Support

RSES $=$ Rosenberg Self-Esteem Scale

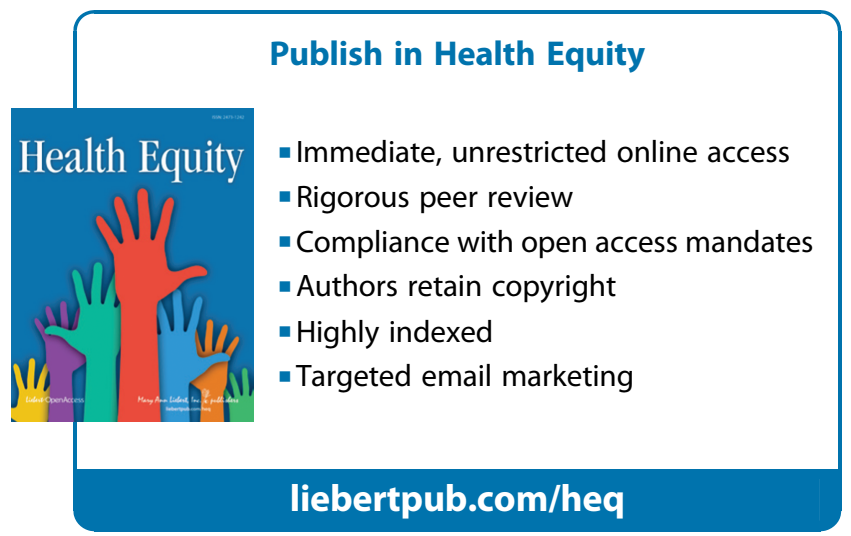

